\title{
Carotenoid consumption is related to lower lipid oxidation and DNA damage in middle-aged men
}

\author{
P. G. Cocate ${ }^{1}$, A. J. Natali ${ }^{2}$, R. C. G. Alfenas ${ }^{1}$, A. de Oliveira ${ }^{3}$, E. C. dos Santos ${ }^{4}$ \\ and H. H. M. Hermsdorff ${ }^{1}$ * \\ ${ }^{1}$ Department of Nutrition and Health, Universidade Federal de Viçosa, Avenue PH Rolfs, Viçosa, \\ Minas Gerais 36571-900, Brazil \\ ${ }^{2}$ Department of Physical Education, Universidade Federal de Viçosa, Viçosa, Minas Gerais, Brazil \\ ${ }^{3}$ Department of Physical Education Science and Health, Universidade Federal de São João del-Rei, \\ São João del-Rei, Minas Gerais, Brazil \\ ${ }^{4}$ Department of General Biology, Universidade Federal de Viçosa, Viçosa, Minas Gerais, Brazil \\ (Submitted 28 November 2014 - Final revision received 11 April 2015 - Accepted 14 April 2015 - First published online 16 June 2015)
}

\section{Abstract}

The present cross-sectional study assessed the potential relationships of carotenoid intake with lipid and oxidative stress markers in middle-aged men. A total of 296 apparently healthy middle-aged men (mean age 50.5 (SD 5.0) years, BMI $25.8\left(\mathrm{sD} 3.5\right.$ ) $\mathrm{kg} / \mathrm{m}^{2}$ ) were recruited to participate in the study. Dietary intake, anthropometry, blood pressure, lifestyle features, blood and urine biomarkers were assessed using validated procedures. The lipid markers included NEFA, Castelli index, and TAG:HDL ratio; oxidative stress markers included urinary 8-hydroxy-2'-deoxyguanosine (8-OHdG), 8-iso-PGF2 $\alpha$ and plasma oxidised-LDL (ox-LDL). We observed a significant inverse association $(P<0.05)$ between NEFA concentrations and consumption of lutein plus zeaxanthin, $\beta$-carotene, $\alpha$-carotene and total carotenoid, while Castelli index was negatively associated with daily intake of lycopene, $\beta$-carotene and total carotenoids. Regarding oxidative stress biomarkers, urinary $8-\mathrm{OHdG}$ and ox-LDL concentrations were also inversely associated $(P<0.05)$ with consumption of lycopene, lutein plus zeaxanthin, $\beta$-carotene, $\alpha$-carotene and total carotenoids, regardless of confounding variables. Moreover, there was a negative association of urinary 8 -iso-PGF $2 \alpha$ concentration with dietary lutein plus zeaxanthin $(\beta-0 \cdot 135$, 95\% CI $-0 \cdot 268$, $-0 \cdot 001), \beta$-carotene $(\beta-0.156,95 \% \mathrm{CI}-0 \cdot 277,-0.034)$ and with the sum of all carotenoids $(\beta-0 \cdot 189,95 \% \mathrm{CI}-0 \cdot 333,-0 \cdot 046)$. In conclusion, total daily carotenoid intake based on five investigated carotenoid types ( $\beta$-cryptoxanthin, lycopene, lutein plus zeaxanthin, $\beta$-carotene and $\alpha$-carotene) was inversely associated with relevant lipid and oxidative stress markers in middle-aged men, with emphasis on $\beta$-carotene that was negatively associated with five of the six lipid and oxidative stress markers evaluated in the present study.

Key words: Food habits: Carotenoids: Lipids: Oxidative stress

Diseases of the circulatory system and cancer are among the leading causes of death in Brazilian men according to the last Brazilian mortality database (2012). It is estimated that nearly one quarter of deaths caused by these diseases affect adult men aged between 40 and 59 years $^{(1)}$. Eating habits can have a significant influence in the development of most chronic diseases, affecting peoples' health throughout their lifetime ${ }^{(2)}$. Thus, the adoption of a healthy diet, including fruits and vegetables, can play an important role in preventing these diseases in middle-aged individuals, since the consumption of these foods have been inversely associated with chronic diseases ${ }^{(3,4)}$ and oxidative stress ${ }^{(5,6)}$.
Fruits and vegetables are sources of nutritional components with high antioxidant capacity such as carotenoids ${ }^{(7)}$, which have the power of singlet oxygen quenchers, and are scavengers of a variety of reactive oxygen species that can be involved in modern chronic disease manifestation ${ }^{(8)}$. Among over 600 carotenoids naturally identified $^{(9)}$, the $\beta$-carotene, $\beta$-cryptoxanthin, $\alpha$-carotene, lycopene and lutein plus zeaxanthin are the major ones found in human plasma or serum $^{(10)}$. Regarding fat-soluble compounds, the lycopene was considered as having the strongest singlet oxygenquenching ability, followed by $\alpha$-carotene, $\beta$-carotene, zeaxanthin, lutein and $\beta$-cryptoxanthin ${ }^{(11)}$. Furthermore,

Abbreviations: HDL-C, HDL-cholesterol; MetS, metabolic syndrome; ox-LDL, oxidised-LDL.

*Corresponding author: H. H. M. Hermsdorff, fax +55 31 3899-2541, email helenhermana@ufv.br 
carotenoids can affect transcription factors (NF- $\mathrm{B}$ or nuclear erythroid-2-related factor 2) and their downstream targets, promoting health benefits by reducing oxidative stress and inflammation $^{(12)}$.

Thus, the antioxidant and anti-inflammatory properties of carotenoids may prevent lipid oxidation and minimise cardiometabolic risks ${ }^{(13)}$. In fact, carotenoids have also been associated with lower concentrations of inflammatory markers $^{(14)}$, and lower chronic disease risks such as the metabolic syndrome (MetS) ${ }^{(15,16)}, \mathrm{CVD}^{(17,18)}$ and cancer ${ }^{(19)}$. However, to our knowledge, the association of carotenoid intake with lipid and oxidative stress markers in apparently healthy subjects has not been clarified yet.

Therefore, we investigated the relationship of carotenoid consumption with lipid and oxidative stress markers in middle-aged men. We hypothesised that there is an inverse association of carotenoids consumption with lipid and oxidative stress biomarkers.

\section{Subjects and methods}

\section{Participants}

The present cross-sectional study was carried out between March and December 2011. The sample size was calculated ${ }^{(20)}$ in February 2011, considering the total number of male staff (1744 individuals), aged between 40 and 59 years, at the Universidade Federal de Viçosa, Viçosa, Brazil, 95\% confidence level, $24.4 \%$ expected prevalence of metabolic condition highly related with oxidative stress ${ }^{(21)}$ (MetS) and $4.5 \%$ sampling error.

We excluded from the study participants with self-reported body weight alterations greater than $3 \mathrm{~kg}$ in the 3 months preceding the study; altered levels of physical activity and eating habits in the 3 months preceding the study; thyroid diseases, heart failure, cerebrovascular diseases, infectious diseases, inflammatory diseases, diseases of the gastrointestinal tract, liver disease, chronic kidney disease and/or a history of kidney stones and cancer in the previous 10 years; eating disorders (anorexia and bulimia) and food allergies. Individuals using vitamin supplements, those using diuretics or drugs that alter food intake and/or the metabolism of nutrients, those with pacemakers and/or prosthetic limbs and elite athletes were also excluded from the study.

The sample size calculated for this study was of 293 participants. This sample size was increased by $2.5 \%$ of subjects (seven individuals) to allow for possible withdrawals from the study. Then, we selected a total of 300 men based on inclusion criteria using systematic sampling. Four subjects who did not answer the FFQ were excluded later. Therefore, the final sample comprised 296 subjects.

The present study was conducted according to the guidelines laid down in the Declaration of Helsinki, and all procedures involving human subjects were approved by the Ethics Committee on Human Research of the Universidade Federal de Viçosa (Reference $n^{\circ}$ 069/2010). Written informed consent was obtained from all subjects.

\section{Dietary and lifestyle data assessment}

An FFQ developed for the Brazilian population ${ }^{(22)}$, which was adapted and published by Queiroz ${ }^{(23)}$, was used to assess the usual dietary intake of the participants of the present study, taking into account their habitual intake in the previous 6 months. We evaluated 105 items from the following foodgroups: milk and dairy products, fats, breads and bread substitutes, cereals, fruits, legumes, vegetables, meats, eggs, beverages and sweets. The FFQ was administered once to each participant between March and December 2011 (study period). Daily food intake was estimated as frequency $x$ portion $\times$ size for each consumed food item. Nutrient intakes including carotenoid intake ( $\beta$-cryptoxanthin, lycopene, lutein plus zeaxanthin, $\beta$-carotene and $\alpha$-carotene) were assessed using the United States Department of Agriculture table ${ }^{(24)}$, since these data are not included in Brazilian food tables. The foods in the FFQ which were not listed in the United States Department of Agriculture table were estimated considering appropriate values of foods that had similar nutritional composition and were prepared using similar cooking methods. Intake of each nutrient was evaluated by a standard spreadsheet software (Excel 2003; Microsoft Corporation).

Data about lifestyle factors, including work position and current smoking status, were collected using a questionnaire administered by a trained interviewer. The subjects of the present study occupied technical/administrative positions, classified as levels A, B, C, D, and E, or were professors. They were divided into two groups according to their education level and work positions: the first group ABC was composed of technical and administrative staff, levels A, B and $\mathrm{C}$, indicating an education level up to high school; the second group DEprof was composed of technical and administrative staff of two levels D and E, and the abbreviation 'prof' stood for professors; subjects in this second group had all completed graduation or post-graduation. Participants were also categorised as current smokers and non-smokers. Finally, habitual physical activity was estimated by the mean number of daily steps (seven consecutive days) measured by a DigiWalker SW-200 pedometer (Yamax Corporation), according to instructions previously described ${ }^{(25)}$.

\section{Anthropometric and biomedical data collection}

Anthropometric measurements such as weight, height, and waist circumference as well as systolic and diastolic blood pressures were taken using standard measurement procedures $^{(25)}$. BMI was calculated as weight $(\mathrm{kg})$ divided by height squared $\left(\mathrm{m}^{2}\right)$. Total body fat percentage was determined by total body scanning with a dual energy X-ray absorptiometry (enCORE software version 13.31; GE/Lunar) in accordance with the manufacturer's instructions.

A venous blood sample was taken after a 12-h overnight fast for measuring total cholesterol, HDL-cholesterol (HDL-C), TAG, NEFA and oxidised-LDL (ox-LDL). Serum total cholesterol, HDL-C and TAG were determined by the enzymatic colorimetric method (Cobas Mira Plus; Roche Diagnostics $\mathrm{GmbH}$ ). NEFA concentration was determined by the kinetic 
Table 1. Anthropometric, clinical and lifestyle characteristics of participants

(Mean values and standard deviations, $n$ 296)

\begin{tabular}{|c|c|c|}
\hline Variables & Mean & SD \\
\hline Age (years) & $50 \cdot 5$ & $5 \cdot 0$ \\
\hline BMI $\left(\mathrm{kg} / \mathrm{m}^{2}\right)$ & $25 \cdot 8$ & 3.5 \\
\hline Total body fat (\%) & $22 \cdot 7$ & $7 \cdot 2$ \\
\hline $\mathrm{HDL}-\mathrm{C}(\mathrm{mmol} / \mathrm{l})$ & $1 \cdot 21$ & 0.33 \\
\hline TAG (mmol/l) & $1 \cdot 61$ & 1.07 \\
\hline NEFA $(\mathrm{mmol} / \mathrm{l})$ & 0.83 & 0.44 \\
\hline 8-OHdG (ng/mg creatinine) & $8 \cdot 40$ & 3.18 \\
\hline 8-iso-PGF2 $\alpha$ (ng/mg creatinine) & 1.49 & 1.54 \\
\hline ox-LDL (U/I) & $55 \cdot 6$ & $16 \cdot 8$ \\
\hline Habitual physical activity (steps/d) & 11099 & 3896 \\
\hline \multicolumn{3}{|l|}{ Work position ABC } \\
\hline$n$ & \multicolumn{2}{|c|}{196} \\
\hline$\%$ & \multicolumn{2}{|c|}{$66 \cdot 2$} \\
\hline \multicolumn{3}{|l|}{ Current smoker } \\
\hline$n$ & \multicolumn{2}{|c|}{40} \\
\hline$\%$ & \multicolumn{2}{|c|}{13.5} \\
\hline
\end{tabular}

HDL-C, HDL-cholesterol; 8-OHdG, 8-hydroxy-2'-deoxyguanosine; ox-LDL, oxidised LDL; Work position ABC, technical-administrative positions $A, B$ or $C$.

spectrophotometry method (EnzyChromFree Fatty Acid Assay; Bioassay Systems), while ox-LDL was assessed by the commercially available ELISA kit (Mercodia) based on the direct sandwich technique.

Castelli index (determined by the ratio between total cholesterol and HDL-C) ${ }^{(26}$ ) and TAG:HDL-C ratio ${ }^{(27)}$, both recognized as atherogenic markes ${ }^{(28,29)}$, were calculated. The MetS was diagnosed by the Alberti et al. ${ }^{(30)}$ criteria.

In turn, urine was collected in sterile tubes (after a 12-h overnight fasting) for measuring 8 -iso-PGF2 $\alpha$ and $8-\mathrm{OHdG}$, the biomarkers of oxidative stress.

Competitive ELISA was used to determine urinary concentrations of 8-iso-PGF2 $\alpha$ (Oxford) and 8-OHdG (Cayman). The analyses were performed according to the manufacturers' instructions. Although the Cayman's kit recognises the 8-OHdG from DNA, the ELISA values are always higher than LC/MS inasmuch as this method also detects 8-hydroxyguanosine and 8-hydroxyguanine from either DNA or RNA. The values of urinary 8 -iso-PGF2 $\alpha$ and 8 -OHdG were normalised by $\mathrm{mg}$ of urinary creatinine, measured by a kinetic colorimetric method, using a Bioclin commercial kit (Cobas Mira Plus; Roche Diagnostics GmbH).

\section{Statistical analyses}

Normal distribution of the data was assessed by the Shapiro-Wilk test. Non-normally distributed variables were log-transformed before statistical analysis. $\beta$-Cryptoxanthin, lycopene, lutein plus zeaxanthin, $\beta$-carotene and $\alpha$-carotene intakes and the sum of these carotenoids consumption was adjusted by daily energy intake using the residual method ${ }^{(31)}$ before statistical analysis. To evaluate the association of these antioxidant compounds with lipid and oxidative stress markers, we used linear multiple regressions, controlled by habitual physical activity (steps/d), work position, daily energy intake (kcal/d), total body fat (percentage) and current smoker status (yes/no).
A stepwise multiple regression analysis was also used to identify the key food-groups and food items from relevant consumptions by the participants that explained carotenoid intake. The participants were then categorised into tertiles based on five investigated carotenoids ( $\beta$-cryptoxanthin, lycopene, lutein plus zeaxanthin, $\beta$-carotene and $\alpha$-carotene). Subsequently, as a complementary analysis, we used the $\chi^{2}$ test for linear trend to compare proportions among total carotenoid consumption and MetS.

Energy consumption outliers were defined as previously described ${ }^{(32)}$. Data processing and analysis were performed using the software STATA version 9.1 (Stata Corporation), considering $P<0.05$.

\section{Results}

Anthropometric, clinical and lifestyle characteristics of the study participants are shown in Table 1. Mean total carotenoids consumed by the study subjects was 7500 (SD 5435) $\mu \mathrm{g} / \mathrm{d}$. Regarding the food-groups, we found that the mean fruit and vegetable intake corresponded to $310.9 \mathrm{~g} / \mathrm{d}$, and that subjects consumed $54.1 \%$ of their total energy from carbohydrate, $27.5 \%$ from fat and $18.4 \%$ from protein (Table 2).

Interestingly, of all food-groups analysed, only fruits, vegetables and eggs explained the variation in carotenoid intake in the present study. Then, we assessed other stepwise multiple regressions with fruit and vegetable items and eggs to verify which items contributed most to the consumption of antioxidants. Thus, the items described in Table 3 accounted for $88.2 \%$ of the total variability in antioxidant carotenoids. When each carotenoid was individually analysed, we also verified that fruit and vegetable food-groups contributed most to the variation in intake than did others. The items

Table 2. Carotenoids, food-groups and macronutrients consumed by participants

(Mean values and standard deviations, $n$ 296)

\begin{tabular}{|c|c|c|}
\hline Variables & Mean & SD \\
\hline$\beta$-Cryptoxanthin $(\mu \mathrm{g} / \mathrm{d})$ & 262 & $302 \cdot 2$ \\
\hline Lycopene $(\mu \mathrm{g} / \mathrm{d})$ & 1597 & $1457 \cdot 3$ \\
\hline Lutein plus zeaxanthin $(\mu \mathrm{g} / \mathrm{d})$ & 2307 & $1910 \cdot 4$ \\
\hline$\beta$-Carotene $(\mu \mathrm{g} / \mathrm{d})$ & 2724 & $2323 \cdot 8$ \\
\hline$\alpha$-Carotene $(\mu \mathrm{g} / \mathrm{d})$ & 610 & $659 \cdot 8$ \\
\hline Total carotenoids $(\mu \mathrm{g} / \mathrm{d})$ & 7500 & $5435 \cdot 0$ \\
\hline Milk and dairy products $(\mathrm{g} / \mathrm{d})$ & $150 \cdot 6$ & $156 \cdot 0$ \\
\hline Breads and bread substitutes (g/d) & $81 \cdot 8$ & $64 \cdot 1$ \\
\hline Fats $(g / d)$ & 5.5 & 6.9 \\
\hline Cereals $(g / d)$ & $168 \cdot 3$ & $84 \cdot 0$ \\
\hline Fruits $(\mathrm{g} / \mathrm{d})$ & $227 \cdot 4$ & $236 \cdot 9$ \\
\hline Meat $(\mathrm{g} / \mathrm{d})$ & $137 \cdot 8$ & 63.52 \\
\hline Legumes $(g / d)$ & $110 \cdot 5$ & $51 \cdot 4$ \\
\hline Vegetables $(\mathrm{g} / \mathrm{d})$ & & $76 \cdot 3$ \\
\hline Soft drink and artificial juice (g/d) & $69 \cdot 8$ & $89 \cdot 4$ \\
\hline Sweets $(g / d)$ & $200 \cdot 7$ & 231.4 \\
\hline \multicolumn{3}{|l|}{ Total energy } \\
\hline $\mathrm{kJ} / \mathrm{d}$ & 6363.4 & $2058 \cdot 5$ \\
\hline $\mathrm{kcal} / \mathrm{d}$ & $1520 \cdot 9$ & $492 \cdot 0$ \\
\hline Carbohydrate (g/d) & $205 \cdot 7$ & $70 \cdot 0$ \\
\hline Lipids (g/d) & 46.5 & $21 \cdot 3$ \\
\hline Protein (g/d) & 69.9 & 21.9 \\
\hline
\end{tabular}


Table 3. Main fruit and vegetable items consumed by participants that contribute to carotenoids consumption ( $\beta$-cryptoxanthin + lycopene + lutein plus zeaxanthin $+\beta$-carotene $+\alpha$-carotene $)^{*}$

\begin{tabular}{lcc}
\hline Fruit and vegetables & $R^{2}$ & Cumulative $R^{2}$ \\
\hline Carrot/pumpkin & 0.610 & 0.610 \\
Dark green leaves (watercress/kale/spinach) & 0.160 & 0.770 \\
Watermelon/melon & 0.040 & 0.810 \\
Papaya & 0.030 & 0.840 \\
Orange & 0.020 & 0.860 \\
Tomatoes & 0.010 & 0.870 \\
Other fruits (grapes/pineapple/pear) & 0.004 & 0.874 \\
Cabbage/cauliflower/broccoli & 0.004 & 0.878 \\
Chayote/zucchini/okra & 0.002 & 0.880 \\
Beets & 0.002 & 0.882 \\
\hline
\end{tabular}

*Banana, apple, lettuce, cucumber and eggs were excluded from stepwise regression model.

from these food-groups accounted for 99.3, 94.2, 55.3, 92.4 and $98.5 \%$ of the total variability in $\beta$-cryptoxanthin, lycopene, lutein plus zeaxanthin, $\beta$-carotene and $\alpha$-carotene consumption, respectively. We observed that papaya $\left(R^{2} 0.62\right)$ contributed to the highest $\beta$-cryptoxanthin intake; watermelon/melon $\left(R^{2} 0 \cdot 49\right)$ favoured the highest lycopene intake; dark green leaves $\left(R^{2} 0 \cdot 40\right)$ supplied the highest lutein plus zeaxanthin intake; carrot/pumpkin $\left(R^{2} 0.76\right.$ and 0.98$)$ facilitated the highest $\beta$-carotene and $\alpha$-carotene intake, respectively.

In terms of lipid markers, there was a tendency $(P=0.079)$ and a negative association $(P<0.05)$ between NEFA concentration and lycopen, lutein plus zeaxanthin, $\beta$-carotene and $\alpha$-carotene, and with the total carotenoid intake, respectively. We observed a significant inverse association of Castelli index values with lycopene, $\beta$-carotene and with the sum of all carotenoids consumed. The oxidative stress biomarkers of urinary 8-OHdG and ox-LDL concentrations were also inversely associated with lycopene, lutein plus zeaxanthin, $\beta$-carotene, $\alpha$-carotene and total carotenoid consumption, regardless of confounding variables. Moreover, there was a negative association of urinary 8 -iso-PGF $2 \alpha$ concentration with lutein plus zeaxanthin, $\beta$-carotene and the sum of all carotenoids consumed (Table 4).

The inclusion of the covariates 'vitamin C consumption' and 'fibre consumption' in the linear regression model did not lead to a relevant change in statistical significance. Surprisingly, when we included 'total fat intake' as a covariate, we found no significant changes either in the statistical outcome (data not shown).

We performed a complementary analysis and observed a significant $(P=0.038)$ lower presence of MetS (19.0\%) among those subjects who consumed higher quantities of total carotenoids (third tertile $=8250 \mu \mathrm{g} / \mathrm{d}$ ), compared to those who consumed lower quantities $(21.4 \%$, second tertile $=4850-8250 \mu \mathrm{g} / \mathrm{d}$ and $31.6 \%$, first tertile $=4850 \mu \mathrm{g} / \mathrm{d}$ ).

\section{Discussion}

The hypothesis of the present cross-sectional study was confirmed, as we observed an inverse association of daily carotenoid intake with lipid and oxidative stress markers. In fact, carotenoids are effective antioxidants which can reduce oxidative stress $^{(8)}$. In this sense, fruit and vegetable intakes explained most of the variations in carotenoid consumption in the present study, confirming that these food types are the major sources of carotenoids ${ }^{(33)}$, and that they lead to lower oxidative stress marker concentrations ${ }^{(5)}$.

Another important finding of the present study was the negative association between NEFA concentrations and total carotenoid consumption. NEFA concentrations have been found to be positively associated with insulin resistance and the occurrence of MetS in another study ${ }^{(34)}$. In this context, we observed lower presence of MetS among those subjects who consumed higher quantities of total carotenoids, compared to those who consumed lower quantities. This result is in accordance with a previous cross-sectional study that observed an inverse association between total carotenoid intake ( $\beta$-cryptoxanthin, lycopene, lutein plus zeaxanthin, $\beta$-carotene and $\alpha$-carotene consumption) and the presence of MetS in middle-aged and elderly men ${ }^{(16)}$.

We also showed that lycopene and $\beta$-carotene consumption were negatively related with Castelli index. In fact, lycopene appears to have hypocholesterolemic effect probably due to the inhibition of activity and expression of the 3-hydroxy-3methylglutaryl coenzyme A reductase enzyme. This leads to a decrease in cholesterol synthesis, modulation of LDL receptor activity and inhibition of the acyl-coenzyme A:cholesterol acyltransferase enzyme activity ${ }^{(35)}$. Furthermore, $\beta$-carotene supplementation $(0 \cdot 2 \%)$ for 6 weeks led to a decrease in serum total cholesterol and non-HDL-C, as well as an increase in total lipid and cholesterol contents excreted in the faeces of female Fisher rats fed with a hypercholesterolemic diet, as compared to rats not supplemented with $\beta$-carotene ${ }^{(36)}$. These mechanisms can partially explain the inverse association between lycopene and $\beta$-carotene consumption and lipid markers observed in the present study.

We also found an inverse association between the oxidative stress biomarkers (urinary 8-OHdG, urinary 8-iso-PGF2 $\alpha$ and ox-LDL concentration) and the consumption of all carotenoids (sum of $\beta$-cryptoxanthin, lycopene, lutein plus zeaxanthin, $\beta$-carotene and $\alpha$-carotene) in the present study. In a prospective 15-year follow-up study, the sum of circulating carotenoids $(\beta$-carotene, $\alpha$-carotene, $\beta$-cryptoxanthin, lycopene, lutein and zeaxanthin) in men and women in the year zero was inversely associated with $\mathrm{F}_{2}$-isoprostane concentration (a recognised oxidative stress marker), and positively associated with the activity of superoxide dismutase (an antioxidant enzyme) in the year $15^{(37)}$. In accordance, an investigation also involving the participants of the Cardiovascular Risk Development in Young Adults (CARDIA) Study found an inverse relationship of the circulating carotenoid concentration ( $\beta$-carotene, $\alpha$-carotene, $\beta$-cryptoxanthin, lutein and zeaxanthin) with serum $\gamma$-glutamyltransferase activity (related to oxidative stress), both cross-sectionally and longitudinally ${ }^{(38)}$.

Interestingly, when we analysed the consumption of each carotenoid, we obtained an inverse association with at least two of the oxidative stress biomarkers assessed, except for 
Table 4. Multiple linear regression models with the carotenoids consumption as the main independent variable

( $\beta$ Coefficients and $95 \%$ confidence intervals)

\begin{tabular}{|c|c|c|c|c|}
\hline Dependent variables & $\beta$ & $95 \% \mathrm{Cl}$ & $R^{2}$ & $P^{\star}$ \\
\hline \multicolumn{5}{|l|}{$\beta$-Cryptoxanthin } \\
\hline NEFA (mmol/l) & -0.015 & $-0.046,0.017$ & 0.11 & 0.358 \\
\hline TAG:HDL ratio & -0.015 & $-0.065,0.034$ & 0.25 & 0.535 \\
\hline Castelli index & -0.008 & $-0.029,0.013$ & 0.19 & 0.456 \\
\hline 8-OHdG (ng/mg Crn) & -0.014 & $-0.042,0.013$ & 0.06 & 0.317 \\
\hline 8-iso-PGF2 $\alpha$ (ng/mg Crn) & -0.024 & $-0.088,0.040$ & 0.08 & 0.463 \\
\hline ox-LDL (U/I) & -0.378 & $-1.699,0.944$ & 0.08 & 0.574 \\
\hline \multicolumn{5}{|l|}{ Lycopene } \\
\hline NEFA (mmol/l) & -0.039 & $-0.082,0.004$ & 0.12 & 0.079 \\
\hline TAG:HDL ratio & -0.058 & $-0.126,0.010$ & 0.25 & 0.095 \\
\hline Castelli index & -0.034 & $-0.063,-0.005$ & 0.20 & 0.023 \\
\hline 8-OHdG (ng/mg Crn) & -0.077 & $-0.115,-0.040$ & 0.11 & $<0.001$ \\
\hline 8-iso-PGF2 $\alpha$ (ng/mg Crn) & -0.063 & $-0.152,-0.025$ & 0.08 & 0.160 \\
\hline ox-LDL (U/l) & -1.903 & $-3.730,-0.075$ & 0.09 & 0.041 \\
\hline \multicolumn{5}{|l|}{ Lutein plus zeaxanthin } \\
\hline NEFA (mmol/l) & -0.099 & $-0.163,-0.035$ & 0.14 & 0.003 \\
\hline TAG:HDL ratio & -0.029 & $-0.131,0.073$ & 0.25 & 0.575 \\
\hline Castelli index & -0.039 & $-0.083,0.004$ & 0.20 & 0.078 \\
\hline 8-OHdG (ng/mg Crn) & -0.082 & $-0.139,-0.025$ & 0.08 & 0.005 \\
\hline 8-iso-PGF2 $\alpha$ (ng/mg Crn) & -0.135 & $-0.268,-0.001$ & 0.09 & 0.048 \\
\hline ox-LDL (U/l) & -2.841 & $-5.585,-0.097$ & 0.09 & 0.043 \\
\hline \multicolumn{5}{|l|}{$\beta$-Carotene } \\
\hline NEFA (mmol/l) & -0.135 & $-0.193,-0.078$ & 0.17 & $<0.001$ \\
\hline TAG:HDL ratio & -0.068 & $-0.161,0.025$ & 0.25 & 0.154 \\
\hline Castelli index & -0.048 & $-0.088,-0.008$ & 0.20 & 0.018 \\
\hline 8-OHdG (ng/mg Crn) & -0.100 & $-0.152,-0.048$ & $0 \cdot 10$ & $<0.001$ \\
\hline 8-iso-PGF2 $\alpha$ (ng/mg Crn) & -0.156 & $-0.277,-0.034$ & 0.09 & 0.012 \\
\hline ox-LDL (U/I) & $-4 \cdot 109$ & $-6.599,-1.620$ & 0.11 & 0.001 \\
\hline \multicolumn{5}{|l|}{$\alpha$-Carotene } \\
\hline NEFA (mmol/l) & -0.058 & $-0.096,-0.019$ & 0.14 & 0.003 \\
\hline TAG:HDL ratio & -0.019 & $-0.080,0.042$ & 0.25 & 0.539 \\
\hline Castelli index & -0.016 & $0.042,0.010$ & 0.19 & 0.240 \\
\hline 8-OHdG (ng/mg Crn) & -0.058 & $-0.092,-0.025$ & 0.09 & 0.001 \\
\hline 8-iso-PGF2 $\alpha$ (ng/mg Crn) & -0.065 & $-0.144,0.014$ & 0.08 & 0.108 \\
\hline ox-LDL (U/I) & -2.439 & $-4.058,-0.821$ & 0.11 & 0.003 \\
\hline \multicolumn{5}{|l|}{ Total carotenoids } \\
\hline NEFA (mmol/l) & -0.138 & $-0.207,-0.069$ & $0 \cdot 16$ & $<0.001$ \\
\hline TAG:HDL ratio & -0.087 & $-0.197,0.023$ & 0.25 & 0.122 \\
\hline Castelli index & -0.059 & $-0.106,-0.012$ & 0.20 & 0.014 \\
\hline 8-OHdG (ng/mg Crn) & -0.135 & $-0.195,-0.074$ & 0.12 & $<0.001$ \\
\hline 8-iso-PGF2 $\alpha$ (ng/mg Crn) & -0.189 & $-0.333,-0.046$ & 0.10 & 0.010 \\
\hline ox-LDL (U/I) & -4.353 & $-7.285,-1.420$ & 0.11 & 0.004 \\
\hline
\end{tabular}

8-OHdG, 8-hydroxy-2'-deoxyguanosine; Crn, creatinine; ox-LDL, oxidised LDL.

${ }^{\star}$ Model adjusted for habitual physical activity (steps/d), work position, daily energy intake (kcal/d), total body fat (\%) and current smoker (yes/no).

$\beta$-cryptoxanthin consumption. In this sense, $\beta$-carotene intake was also negatively associated with 8-iso-PGF2 $\alpha$ among elderly men in a cohort study ${ }^{(39)}$. Additionally, plasma $\beta$-carotene concentration was the most important determinant of serum LDL conjugated dienes (an indicator of circulating ox-LDL) variation, which was shown to be the most efficient antioxidant among the serum carotenoids in adult men in another cohort study ${ }^{(40)}$. The authors of a study involving patients with coronary artery disease and apparently healthy subjects (control) verified a negative correlation of plasma $\alpha$ - and $\beta$-carotene concentration with the susceptibility of DNA to oxidation ${ }^{(41)}$. In turn, in a randomised cross-over study, lycopene supplementation in three different treatments $(39 \cdot 2 ; 50.4$ and $75.0 \mathrm{mg} / \mathrm{d}$ of lycopene) for a period of a week also led to a significant reduction in
LDL oxidation (measured by LDL-thiobarbituric acid-reactive substance and LDL conjugated diene) in healthy adults ${ }^{(42)}$. On the other hand, daily lycopene supplementation (30 mg) for 8 weeks reduced lymphocyte DNA damage and urinary 8-OHdG in healthy adult human subjects in a double-blind, placebo-controlled intervention trial ${ }^{(43)}$.

The inverse relationship of carotenoids with oxidative stress biomarkers have been observed in in vitro studies as well. For instance, Kameji et al. ${ }^{(44)}$ observed higher reactive oxygen species levels (assessed by oxidation of $2^{\prime}, 7^{\prime}$-dichlorodihydrofluorescein diacetate) in 3T3L1 adipocytes pretreated with TNF- $\alpha$ in the absence of $\beta$-carotene than in untreated cells, while those cells pretreated with $\beta$-carotene had no induction of dichlorodihydrofluorescein diacetate. Thus, they showed that $\beta$-carotene accumulation is able to restore reactive 
oxygen species levels in insulin-resistant adipocytes.Also in another study on human umbilical vein endothelial cells, $\beta$-carotene and lycopene affected the inflammation and oxidation induced by TNF- $\alpha$, through the inhibition of the expression of adhesion molecules, and suppression of the increase in reactive oxygen species generation and of nitrotyrosine (index of $\mathrm{ONOO}^{-}$formation) intracellular levels, which led to augmented NO levels and bioavailability ${ }^{(45)}$. Therefore, in a pro-oxidant condition, physiological concentrations of $\beta$-carotene and lycopene can reduce the inflammatory activity, which can act positively on vascular inflammatory states probably by increasing vascular NO bioavailability ${ }^{(45)}$. Moreover, the supplementation of $\beta$-carotene and lutein had an inhibitory effect on $\mathrm{H}_{2} \mathrm{O}_{2}$ (reative oxygen species)-induced activation of NF- $\mathrm{KB}$ in human gastric epithelial cells cultured ${ }^{(46)}$. It suggests the antioxidant capacity of carotenoids and their possible protective effect against chronic diseases induced by oxidative stress.

Additionally, an inverse relationship of carotenoids (i.e. $\beta$-carotene, lycopene and lutein plus zeaxanthin) with the manifestation of some chronic diseases has been verified ${ }^{(47,48)}$ In the present study, we assessed the concentration of oxidative stress biomarkers highly associated with CVD $\left(\mathrm{F}_{2} \text {-isoprostane and } \mathrm{Ox}-\mathrm{LDL}\right)^{(49,50)}$ and cancer $(8-\mathrm{OHdG})^{(51)}$. However, the beneficial role of $\beta$-carotene ${ }^{(52)}$ and lycopene ${ }^{(18)}$ consumption in the prevention of cardiovascular events has not been observed in some studies. This discrepancy may be due to the consumption of excessive doses associated with synthetic carotenoids consumption, which could favour the occurrence of pro-oxidant instead of antioxidant effects ${ }^{(13)}$ and/or the influence of some dietetic and lifestyle variables (e.g. high fat intake, sedentary life style and smoking). Despite this observation, results of the present study indicate the important role of carotenoid intake, mainly those derived from fruits and vegetables, in reducing oxidative stress biomarker concentrations. It also suggests that the assessment of habitual carotenoid consumption may be a useful tool to assess the oxidative stress status in healthy middle-aged men.

It is noteworthy, that our outcomes were independent of relevant environmental (smoking and habitual physical activity) and dietary confounding factors such as vitamin $\mathrm{C}$ and fibre intake, and thus showing the powerful inverse relationship of carotenoid intake with lipid and oxidative stress biomarkers. Thus, these bioactive compounds (carotenoids) may have a promising role in the prevention or control of chronic diseases.

The present study has to acknowledge, however, its limitations. First, given the cross-sectional nature of the investigation, the results must be cautiously considered as they cannot guarantee 'cause and effect' relationship, although we controlled the potential confounding variables as much as possible. Second, borrowing nutrient intake data from the USDA table and applying them to the Brazilian food intake situation might not have truly reflected the research scenario of the present study. Third, it was difficult to take other dietary confounding factors of polyphenols and vitamin $\mathrm{E}$ into account. And fourth, we did not assess serum carotenoid concentrations. However, the present study draws strength from the fact that analyses of carotenoid consumption based on FFQ have been used in previous epidemiological studies ${ }^{(16,53)}$.

\section{Conclusion}

Daily carotenoid intake was cross-sectionally and negatively associated with relevant lipid and oxidative stress markers in the middle-aged men participants of the present study; the results suggest that regular consumption of fruits and vegetables could lead to health benefits, since daily consumption of these bioactive compounds was mostly derived from these food-groups.

\section{Acknowledgements}

The authors thank the participants of the present study from the Universidade Federal de Viçosa, Viçosa, Brazil for their contribution, the nursing staff for excellent technical assistance and the students for untiring help in the fieldwork.

This work was supported by the Foundation for Research Support of the State of Minas Gerais (grant number CDSAPQ-02189-10 and CDS-APQ-02535-13). A. J. N., R. C. G. A., and $\mathrm{H}$. H. M. H. are CNPq fellows.

None of the authors has any conflict of interest to declare.

The authors's contributions are as follows: P. G. C. designed the study, collected and analysed the data, wrote the initial draft and organised the final version of the manuscript; A. d. O. and E. C. d. S. collected the data; A. J. N., R. C. G. A. and H. H. M. H. designed the study, analysed the data and helped in the organisation of the manuscript. All authors assisted in editing the manuscript. All authors read and approved the final version of the manuscript.

\section{References}

1. Ministry of Health's Database (DATASUS) (2015) Health information. Mortality. http://tabnet.datasus.gov.br/cgi/ deftohtm.exe?obitocorr/cnv/obitocorr.def (accessed 13 march 2015).

2. Bressan J, Hermsdorff HH, Zulet MA, et al. (2009) [Hormonal and inflammatory impact of different dietetic composition: emphasis on dietary patterns and specific dietary factors]. Arq Bras Endocrinol Metabol 53, 572-581.

3. Hermsdorff HH, Zulet MA, Puchau B, et al. (2010) Fruit and vegetable consumption and proinflammatory gene expression from peripheral blood mononuclear cells in young adults: a translational study. Nutr Metab (Lond) 7, 42 .

4. Jung SK, Kim K, Tae K, et al. (2013) The effect of raw vegetable and fruit intake on thyroid cancer risk among women: a case-control study in South Korea. Br J Nutr 109, 118-128.

5. Cocate PG, Natali AJ, Oliveira A, et al. (2014) Fruit and vegetable intake and related nutrients are associated with oxidative stress markers in middle-aged men. Nutrition 30, $660-665$.

6. Hermsdorff HH, Barbosa KB, Volp AC, et al. (2012) Vitamin $\mathrm{C}$ and fibre consumption from fruits and vegetables improves oxidative stress markers in healthy young adults. Br J Nutr 107, 1119-1127.

7. Hughes DA (2001) Dietary carotenoids and human immune function. Nutrition 17, 823-827. 
8. Fiedor J \& Burda K (2014) Potential role of carotenoids as antioxidants in human health and disease. Nutrients $\mathbf{6}$, $466-488$.

9. Jomova K \& Valko M (2013) Health protective effects of carotenoids and their interactions with other biological antioxidants. Eur J Med Chem 70, 102-110.

10. Parker RS (1989) Carotenoids in human blood and tissues. J Nutr 119, 101-104.

11. Di Mascio P, Murphy ME \& Sies H (1991) Antioxidant defense systems: the role of carotenoids, tocopherols, and thiols. Am J Clin Nutr 53, 194S-200S.

12. Kaulmann A \& Bohn T (2014) Carotenoids, inflammation, and oxidative stress - implications of cellular signaling pathways and relation to chronic disease prevention. Nutr Res 34, 907-929.

13. Ciccone MM, Cortese F, Gesualdo M, et al. (2013) Dietary intake of carotenoids and their antioxidant and antiinflammatory effects in cardiovascular care. Mediators Inflamm 2013, 782137.

14. Calder PC, Ahluwalia N, Brouns F, et al. (2011) Dietary factors and low-grade inflammation in relation to overweight and obesity. Br J Nutr 106, Suppl. 3, S5-78.

15. Coyne T, Ibiebele TI, Baade PD, et al. (2009) Metabolic syndrome and serum carotenoids: findings of a cross-sectional study in Queensland, Australia. Br J Nutr 102, 1668-1677.

16. Sluijs I, Beulens JW, Grobbee DE, et al. (2009) Dietary carotenoid intake is associated with lower prevalence of metabolic syndrome in middle-aged and elderly men. J Nutr 139, 987-992.

17. Jacques PF, Lyass A, Massaro JM, et al. (2013) Relationship of lycopene intake and consumption of tomato products to incident CVD. Br J Nutr 110, 545-551.

18. Osganian SK, Stampfer MJ, Rimm E, et al. (2003) Dietary carotenoids and risk of coronary artery disease in women. Am J Clin Nutr 77, 1390-1399.

19. Michaud DS, Feskanich D, Rimm EB, et al. (2000) Intake of specific carotenoids and risk of lung cancer in 2 prospective US cohorts. Am J Clin Nutr 72, 990-997.

20. Dean AG, Dean JA, Colombier D, et al. (1994) Epi Info, Version 6: A Word Processing, Database, and Statistics Program for Epidemiology on Microcomputers. Atlanta, GA: Centers for Disease Control and Prevention.

21. Sá NN \& Moura EC (2010) Factors associated with the burden of metabolic syndrome disease among Brazilian adults. Cad Saúde Pública 26, 1853-1862.

22. Ribeiro AB \& Cardoso MA (2002) Development of a food frequency questionnaire as a tool for programs of chronic diseases prevention. Rev Nutr 15, 239-245.

23. Queiroz KC (2008) Dietary profile and nutritional factors associated with glycemic control in children and adolescents with type 1 diabetes. Dissertation, Universidade Federal de Minas Gerais

24. US Department of Agriculture, Agricultural Research Service (2013) USDA National Nutrient Database for Standard Reference, Release 26. http://ndb.nal.usda.gov/ndb/foods (accessed 11 January 2014).

25. Cocate PG, de Oliveira A, Hermsdorff HH, et al. (2014) Benefits and relationship of steps walked per day to cardiometabolic risk factor in Brazilian middle-aged men. J Sci Med Sport 17, 283-287.

26. Castelli WP, Abbott RD \& McNamara PM (1983) Summary estimates of cholesterol used to predict coronary heart disease. Circulation 67, 730-734

27. Gaziano JM, Hennekens CH, O'Donnell CJ, et al. (1997) Fasting triglycerides, high-density lipoprotein, and risk of myocardial infarction. Circulation 96, 2520-2525.
28. da Luz PL, Favarato D, Faria-Neto JR, et al. (2008) High ratio of triglycerides to HDL-cholesterol predicts extensive coronary disease. Clinics (Sao Paulo) 63, 427-432.

29. Malaspina JP, Bussiere H \& Le Calve G (1981) The total cholesterol/HDL cholesterol ratio: a suitable atherogenesis index. Atherosclerosis 40, 373-375.

30. Alberti KG, Eckel RH, Grundy SM, et al. (2009) Harmonizing the metabolic syndrome: a joint interim statement of the International Diabetes Federation Task Force on Epidemiology and Prevention; National Heart, Lung, and Blood Institute; American Heart Association; World Heart Federation; International Atherosclerosis Society; and International Association for the Study of Obesity. Circulation 120, $1640-1645$.

31. Willett WC (1998) Nutritional Epidemiology, 2nd ed New York: Oxford University Press.

32. Cocate PG, Natali AJ, Oliveira A, et al. (2015) Red but not white meat consumption is associated with metabolic syndrome, insulin resistance and lipid peroxidation in Brazilian middle-aged men. Eur J Prev Cardiol 22, 223-230.

33. Maiani G, Caston MJ, Catasta G, et al. (2009) Carotenoids: actual knowledge on food sources, intakes, stability and bioavailability and their protective role in humans. Mol Nutr Food Res 53, Suppl. 2, S194-S218.

34. Athyros VG, Tziomalos K, Karagiannis A, et al. (2011) Dyslipidaemia of obesity, metabolic syndrome and type 2 diabetes mellitus: the case for residual risk reduction after statin treatment. Open Cardiovasc Med J 5, 24-34.

35. Palozza P, Catalano A, Simone RE, et al. (2012) Effect of lycopene and tomato products on cholesterol metabolism. Ann Nutr Metab 61, 126-134.

36. Silva LS, de Miranda AM, de Brito Magalhaes CL, et al. (2013) Diet supplementation with $\beta$-carotene improves the serum lipid profile in rats fed a cholesterol-enriched diet. J Physiol Biochem 69, 811-820.

37. Hozawa A, Jacobs DR Jr, Steffes MW, et al. (2007) Relationships of circulating carotenoid concentrations with several markers of inflammation, oxidative stress, and endothelial dysfunction: the Coronary Artery Risk Development in Young Adults (CARDIA)/Young Adult Longitudinal Trends in Antioxidants (YALTA) study. Clin Chem 53, 447-455.

38. Lee DH, Gross MD, Jacobs DR, et al. (2004) Association of serum carotenoids and tocopherols with $\gamma$-glutamyltransferase: the Cardiovascular Risk Development in Young Adults (CARDIA) study. Clin Chem 50, 582-588.

39. Helmersson J, Arnlov J, Larsson A, et al. (2009) Low dietary intake of $\beta$-carotene, $\alpha$-tocopherol and ascorbic acid is associated with increased inflammatory and oxidative stress status in a Swedish cohort. Br I Nutr 101, 1775-1782.

40. Karppi J, Nurmi T, Kurl S, et al. (2010) Lycopene, lutein and $\beta$-carotene as determinants of LDL conjugated dienes in serum. Atherosclerosis 209, 565-572.

41. Park E, Kyoung Park Y, Kim SM, et al. (2009) Susceptibility to oxidative stress is greater in Korean patients with coronary artery disease than healthy subjects. J Clin Biochem Nutr 45, 341-346.

42. Agarwal S \& Rao AV (1998) Tomato lycopene and low density lipoprotein oxidation: a human dietary intervention study. Lipids 33, 981-984.

43. Devaraj S, Mathur S, Basu A, et al. (2008) A dose-response study on the effects of purified lycopene supplementation on biomarkers of oxidative stress. I Am Coll Nutr 27, $267-273$

44. Kameji H, Mochizuki K, Miyoshi N, et al. (2010) $\beta$-Carotene accumulation in 3T3-L1 adipocytes inhibits the elevation of reactive oxygen species and the suppression of genes related 
to insulin sensitivity induced by tumor necrosis factor- $\alpha$. Nutrition 26, 1151-1156.

45. Di Tomo P, Canali R, Ciavardelli D, et al. (2012) $\beta$-Carotene and lycopene affect endothelial response to TNF- $\alpha$ reducing nitro-oxidative stress and interaction with monocytes. Mol Nutr Food Res 56, 217-227.

46. Kim Y, Seo JH \& Kim H (2011) $\beta$-Carotene and lutein inhibit hydrogen peroxide-induced activation of NF- $\mathrm{B}$ and IL-8 expression in gastric epithelial AGS cells. J Nutr Sci Vitaminol (Tokyo) 57, 216-223.

47. Rao AV \& Rao LG (2007) Carotenoids and human health Pharmacol Res 55, 207-216.

48. Ribaya-Mercado JD \& Blumberg JB (2004) Lutein and zeaxanthin and their potential roles in disease prevention. $J$ Am Coll Nutr 23, 567S-587S.

49. Chen K \& Keaney JF Jr. (2012) Evolving concepts of oxidative stress and reactive oxygen species in cardiovascular disease. Curr Atheroscler Rep 14, 476-483.
50. Schwedhelm E, Bartling A, Lenzen $\mathrm{H}$, et al. (2004) Urinary 8-iso-prostaglandin $\mathrm{F} 2 \alpha$ as a risk marker in patients with coronary heart disease: a matched case-control study. Circulation 109, 843-848.

51. Valavanidis A, Vlachogianni T \& Fiotakis C (2009) 8-Hydroxy-2' -deoxyguanosine (8-OHdG): a critical biomarker of oxidative stress and carcinogenesis. $J$ Environ Sci Health C Environ Carcinog Ecotoxicol Rev 27, 120-139.

52. Ye Y, Li J \& Yuan Z (2013) Effect of antioxidant vitamin supplementation on cardiovascular outcomes: a metaanalysis of randomized controlled trials. PLOS ONE $\mathbf{8}$, e56803.

53. Sahni S, Hannan MT, Blumberg J, et al. (2009) Inverse association of carotenoid intakes with $4-y$ change in bone mineral density in elderly men and women: the Framingham Osteoporosis Study. Am J Clin Nutr 89, $416-424$. 